\title{
Lung Microbiota is Associated with Bacterial Detection and with Clinical Improvements in Severe Community-Acquired Pneumonia Patients
}

\section{Sisi Du}

China-Japan Friendship Hospital

\section{Binbin Li}

China-Japan Friendship Hospital

\section{Yimin Wang}

China-Japan Friendship Hospital

\section{Yudi Xia}

China-Japan Friendship Hospital

\section{Lianhan Shang}

China-Japan Friendship Hospital

\section{Donghao Yu}

China-Japan Friendship Hospital

Zhibo Liu

China-Japan Friendship Hospital

\section{Chunlei Wang}

China-Japan Friendship Hospital

Xinmeng Liu

China-Japan Friendship Hospital

\section{Zhujia Xiong}

China-Japan Friendship Hospital

Xiaohui Zou

China-Japan Friendship Hospital

\section{Binghuai Lu}

China-Japan Friendship Hospital

\section{Yingmei Liu}

China-Japan Friendship Hospital

Bin Cao ( $\nabla$ caobin_ben@163.com )

China-Japan Friendship Hospital

\section{Research}


Keywords: Severe community-acquired pneumonia, lung microbiota, clinical improvements, 7-category ordinal scale, Prevotellaceae

Posted Date: July 9th, 2020

DOI: https://doi.org/10.21203/rs.3.rs-40606/v1

License: (c) (1) This work is licensed under a Creative Commons Attribution 4.0 International License. Read Full License 


\section{Abstract}

Background: Few studies have described the key features of the lung microbiota in patients with severe community-acquired pneumonia (SCAP). We conducted the study to identify the association between the lung microbiota on admission and the clinical prognosis in SCAP patients.

Methods: The consecutive SCAP patients admitted from intensive care unit (ICU) were enrolled prospectively. 16S rRNA gene sequencing was applied to bronchoalveolar lavage fluid (BALF) collected within 48 hours. The clinical information was recorded during the stay of hospitalization. The primary endpoint was the rate of clinical improvements defined as a decrease of 2 categories and above on a 7category ordinal scale within 14 days following bronchoscopy.

Results: Sixty-seven patients were included. Multivariable Permutational multivariate analysis of variance (PERMANOVA) found that bacterial detection had the strongest independent relationship with the lung microbiota $\left(R^{2}=0.033 ; p=0.018\right)$, followed by acute kidney injury (AKI $\left.R^{2}=0.032 ; p=0.011\right)$ and plasma MIP-1 beta level $\left(R^{2}=0.027 ; p=0.044\right)$. Random forest identified that families Prevotellaceae, Moraxellaceae, Staphylococcaceae were the biomarkers related to bacterial detection. In the patients with positive bacteriology results, the mean relative abundance of families Prevotellaceae and Actinomycetaceae decreased while families Moraxellaceae, Staphylococcaceae and Streptococcaceae increased. Multivariable Cox regression showed that the increase in alpha-diversity and the abundance of families Prevotellaceae and Actinomycetaceae were associated with clinical improvements.

Conclusions: The bacterial detection and patients' intrinsic factors were associated with the lung microbiota. The increased alpha diversity and the enrichment of families Prevotellaceae and Actinomycetaceae in the lung microbiota were associated with clinical improvements.

\section{Background}

Patients with severe community acquired pneumonia (SCAP) are usually complicated with hypoxemia, acute kidney injury (AKI), sepsis and required intensive care unit (ICU) care[1, 2]. Although the broadspectrum antibiotic and advanced oxygen support are used commonly in the therapeutic regimen, the death in SCAP patients remains as high as 36\%[3]. Next generation sequencing (NGS) technology has revealed that the human lung contains complex and dynamic microbiota community[4]. Recently, several studies have suggested that the lung microbiota is associated with the respiratory infection and disease outcomes. The murine model proves that the intact microbiota contributes to the protection against bacterial pathogens with GM-CSF signaling[5]. The sputum microbial composition is found to be associated with length of stay and ICU admission in the children hospitalized for CAP[6]. Besides, the enrichment of specific taxon in the airway microbiota, such as Enterobacteriaceae, might be related to high plasma inflammatory cytokine level and the development of acute respiratory distress syndrome (ARDS), and be predictive of fewer ventilator-free days in patients with critical illness[7, 8]. However, few studies have described the key features of the lung microbiota in patients with SCAP. 
A range of factors may contribute to the complexity of the lung microbiota in SCAP patients. The overgrowth of the invasive pneumonia-associated pathogens in patients' lower respiratory tract may cause a decline in the lung microbiota diversity and the microbiota might even be dominated by single species[9]. Based on the new conceptual models of respiratory microbiology proposed by Robert $P$ Dickson, the lung microbiota is determined by three factors: microbial immigration, elimination, and the relative reproduction rates of the members which are affected by the regional growth conditions such as oxygen tension, $\mathrm{pH}$, alveolar ventilation and temperature in the lung[10,11]. Critical illness alters the internal environment and pathophysiology of the respiratory tract of the patients. Laryngeal dysfunction, supine positioning, aspiration or confusion, the ICU management including antibiotic use, mechanical ventilation and vasopressors have impacts on the balance of the immigration and elimination of the airway microorganisms[12]. Collectively, these clinical parameters have the potential to change the lung microbial composition of the SCAP patients.

Therefore, we conducted the study to explore the clinical factors that may be associated with the lung microbiota in patients with SCAP and identify the important taxa which may predict the clinical prognosis.

\section{Methods}

\section{Study populations}

All consecutive severe pneumonia cases admitted from ICU between March 2018 and March 2019 were registered prospectively. The inclusion criteria were (1) age $\geq 18$ years old; (2) diagnosed with SCAP according to the 2007 Infectious Disease Society of America/American Thoracic Society guidelines (Table S1)[13]; (3) The time from onset of illness $\leq 7$ days. The patients whose time of disease less than 14 days while condition exacerbation within 7 days before admission to ICU were also included (Fig. 1). Patients were excluded if they had one of the following criteria: (1) a history of hospitalization within 14 days before illness onset; (2) bronchoscopy couldn't be performed within 48 hours after admission; (3) peripheral blood specimens were not available within 24 hours; (4) pregnancy or breastfeeding; (5) had an alternative diagnosis at the end of the study, including lung cancer, pulmonary tuberculosis or pulmonary embolism.

\section{Sample Collection}

The bronchoscopy was performed at bedside within 48 hours after the patients admitted to ICU using the standard clinical protocol. The bronchoscope was inserted through the nose or orotracheal tube and the bronchoalveolar lavage fluid (BALF) samples were collected. About $15 \mathrm{ml}$ specimen was immediately sent to the microbiology laboratory for routine bacterial, fungal and viral examinations, and the remaining BALF was stored at $-80^{\circ} \mathrm{C}$ until further processing. Blood samples were obtained within 24 hours of ICU arrival. They were centrifuged and the plasma stored at $-80^{\circ} \mathrm{C}$. 


\section{6s Rrna Gene Sequencing}

DNA was extracted from BALF and negative control using the Maxwell ${ }^{\circledR}$ RSC Whole Blood DNA Kit (Promega, USA). The V3-V4 hypervariable region of the 16S rRNA gene of all samples was amplified by polymerase chain reaction (PCR). The pooled library was sequenced on an Illumina Miseq platform (Illumina, San Diego, CA, United States) using pair-end sequencing $(2 \times 300 \mathrm{bp})$. Details were shown in Supplementary Material.

\section{Data Collection}

The following data were collected using a standard case report form: demographic data, underlying diseases, the time of illness onset, clinical symptoms, laboratory findings, microbiology results, radiographic data, antimicrobial use, glucocorticoid use, mechanical ventilation use and so on. The included patients were followed up until they were discharged or died.

\section{Cytokine Measurement}

The Plasma IL-4, IL-6, IL-8, MIP-1 beta, VEGF-A and MMP-9 of the SCAP patients were detected using a magnetic bead-based multiplex immunoassay and read on a Bio-Plex 200 suspension array system (BioRad, Hercules, CA, USA) according to the manufacturer's instructions.

\section{Definition}

The primary endpoint was the rate of clinical improvements, which were defined as a decrease of 2 categories and above on a 7-category ordinal scale within 14 days following bronchoscopy. The ordinal scales which were also used in our influenza and Covid-19 studies consisted of the following categories: 1 , not hospitalized with resumption of normal activities; 2 , not hospitalized, but unable to resume normal activities; 3 , hospitalization, not requiring supplemental oxygen; 4 , hospitalization, requiring supplemental oxygen; 5 , hospitalization, requiring nasal high-flow oxygen therapy and/or noninvasive mechanical ventilation; 6 , hospitalization, requiring extracorporeal membrane oxygenation and/or invasive mechanical ventilation; 7, death[14-16]. Immunocompromised status in our study was defined as the patients having a history of cancer with neutropenia (absolute neutrophil count $<0.5 \times 109 / \mathrm{L}$ ), hematological malignancies, solid malignancies receiving chemotherapy during the previous 3 months, solid organ or bone-marrow transplant, active graft-versus-host disease, bronchiolitis obliterans, human immunodeficiency virus infection, immunoglobulin deficiency, using immunosuppressive agents, or current treatment with systemic corticosteroids ( $\geq 20 \mathrm{mg}$ of prednisone per day or its equivalent) for $>30$ continuous days before illness onset[17]. The presence of ARDS on admission were diagnosed according to the Berlin definition[18]. For the nonventilated subjects who had a history of acute respiratory failure within 7 days because of a known respiratory events and bilateral pulmonary infiltration on chest x-ray 
with PaO2/FiO2 below 300 mmHg, ARDS was also considered. Sepsis, septic shock and acute kidney injury were diagnosed based on the third international consensus and Kidney Disease Improving Global Guidelines (KDIGO) clinical practice guidelines, respectively[19, 20]. Acute cardiac insufficiency was diagnosed by cardiologists based on clinical vitals, laboratory findings and echocardiography. Pneumonia severity was assessed by CURB-65, APACHE-II and PSI risk class. The order of performing bronchoscopy is based the time sequence of signing the bronchoscopy informed consent.

\section{Pathogen Detection}

All the specimens for the microbiology diagnosis were collected within 48 hours after admission. Viral etiology was considered positive if the respiratory virus was detected in sputum, endotracheal aspirates (ETA), BALF, or nasopharyngeal (NP) swabs by real-time PCR (Zhijiang, Shanghai, China), including respiratory syncytial virus, influenza virus types $A$ and $B$, parainfluenza virus, rhinovirus, coronavirus, human metapneumovirus and adenovirus. Bacterial or atypical pathogen detection were considered positive if one of the following criteria was met: (1) positive bacterial culture from blood or pleural fluid; (2) positive urinary antigen for Legionella pneumophila (Binax Now; Trinity Biotech, Bray, Ireland) or Streptococcus pneumoniae (Binax Now; Emergo Europe, Amsterdam, The Netherlands); (3) detection of Mycoplasma pneumoniae or Chlamydia pneumoniae in sputum, BALF, ETA, or NP swabs by real-time PCR(Zhijiang, Shanghai, China); 4) detection of L. pneumophila in sputum, BALF, or ETA by real-time PCR (Zhijiang, Shanghai, China); (5) bacteria with moderate to heavy growth $(>3+$ growth) in qualified sputum or ETA, or quantified culture in BALF of $\geq 104 \mathrm{CFU} / \mathrm{mL}$ [17]. The diagnosis of IFD was based on the revision and update of the consensus definitions of invasive fungal disease[21].

\section{Statistical analysis}

The software VSEARCH (version 2.7.1) and USEARCH version (10.0) were used to process the sequencing data. Reads were denoised into Zero-radius Operational Taxonomic Units (ZOTUs) with UNOISE3. After removal of ZOTUs identified as contaminants with decontam package or observed in the controls (Table S2) and whose relative abundance was less than $0.01 \%$, a total of 490 ZOTUs were analyzed. Statistical analysis was performed in R version 3.6.2 via the Rstudio interface. PERMANOVA (vegan R-package) based on Bray-Curtis distance was performed to assess the association between the clinical factors and the lung microbiota. A random forest learning approach (randomForest R-package) was used to identify the clinical factors-associated taxon. Wilcoxon rank sum test test and generalized linear models (GLM) were performed to compare the relative abundance of the species. Multivariable-adjusted Cox regression (adjusted for sample season, plasma IL-8 level, CURB-65, APACHEIl scores, presence of shock at sampling, oxygen index on admission, creatinine level and microbiology results) was performed to assess the association between lung microbiota and clinical improvements.

\section{Results}




\section{Patients' characteristics and bacterial profiles}

A total of 67 patients were included (Table 1) and the flowchart was shown in Fig. 1. 67 BALF samples from the patients were analyzed. Proteobacteria was the most abundant phylum in the lung microbiota, accounting for $43.7 \%$ of the total filtered reads, followed by Firmicutes (30.94\%), Actinobacteria (7.93\%), Bacteroidetes (4.6\%) and Fusobacteria (1.17\%). At family level, the most abundant taxa were Pseudomonadaceae (23.22\%), Streptococcaceae (13.71\%), Pasteurellaceae (11.33\%), Corynebacteriaceae (5.72\%), Moraxellaceae (5.15\%), Staphylococcaceae (4.94\%), Bacillaceae_1 (4.5\%), Veillonellaceae (2.98\%), Prevotellaceae (2.11\%) and Enterobacteriaceae (1.74\%). 
Table 1

Baseline characteristics of the patients

\begin{tabular}{|c|c|}
\hline Variable & $N=67$ \\
\hline Age, years, median \pm IQR & $64 \pm 22$ \\
\hline Male, n (\%) & $50(74.63)$ \\
\hline \multicolumn{2}{|l|}{ Smoking status ${ }^{a}$} \\
\hline Current smoker, n (\%) & $16(23.88)$ \\
\hline Former smoker, n (\%) & $19(28.36)$ \\
\hline Nonsmoker, n (\%) & $32(47.76)$ \\
\hline Drinking history, n (\%) & $13(19.4)$ \\
\hline Immunocompromised status, n (\%) & $10(14.93)$ \\
\hline Chronic respiratory disease, n (\%) & $11(16.42)$ \\
\hline \multicolumn{2}{|l|}{ Sample season, n (\%) } \\
\hline Spring & $16(23.88)$ \\
\hline Summer & $12(17.91)$ \\
\hline Autumn & $12(17.91)$ \\
\hline Winter & $27(40.30)$ \\
\hline \multicolumn{2}{|l|}{ Symptoms, n (\%) } \\
\hline Fever & $53(79.1)$ \\
\hline Cough & $50(74.63)$ \\
\hline Sputum & $36(53.73)$ \\
\hline Dyspnea & $54(80.60)$ \\
\hline Confusion/disorientation & 15 (22.39) \\
\hline \multicolumn{2}{|l|}{ Laboratory findings on admission } \\
\hline $\mathrm{PH}$, median \pm IQR & $7.443 \pm 0.058$ \\
\hline $\mathrm{PaO}_{2} / \mathrm{FiO}_{2}, \mathrm{mmHg}$, mean $\pm \mathrm{sd}$ & $181.36 \pm 78.62$ \\
\hline Procalcitonin > 1 ng/mL, n (\%) & $36(53.73)$ \\
\hline Creatinine > $106 \mu \mathrm{mol} / \mathrm{L}, \mathrm{n}(\%)$ & $28(41.79)$ \\
\hline Initial radiographic findings, $\mathrm{n}(\%)$ & \\
\hline
\end{tabular}




\begin{tabular}{|c|c|}
\hline Variable & $N=67$ \\
\hline Diffuse bilateral pulmonary infiltration & $56(83.58)$ \\
\hline Pleural effusion & $25(37.31)$ \\
\hline \multicolumn{2}{|l|}{ Pathogen identified ${ }^{b}, \mathrm{n}(\%)$} \\
\hline Viral detection only & $26(38.81)$ \\
\hline Bacterial detection only & $12(17.91)$ \\
\hline Viral-bacterial co-infection & $6(8.96)$ \\
\hline Atypical pathogens ${ }^{c}$ & $9(13.43)$ \\
\hline Probable IFD & $8(11.94)$ \\
\hline \multicolumn{2}{|l|}{ Complications at sampling, $\mathrm{n}(\%)$} \\
\hline ARDS & $29(43.28)$ \\
\hline Sepsis & $65(97.01)$ \\
\hline Septic shock & $21(31.34)$ \\
\hline AKI & $23(34.33)$ \\
\hline Acute cardiac insufficiency & $15(22.39)$ \\
\hline Antibiotic used before sampling ${ }^{\mathrm{d}}, \mathrm{n}(\%)$ & $67(100)$ \\
\hline Carbapenems & $22(32.84)$ \\
\hline Beta-lactams plus fluoroquinolones only & $37(55.22)$ \\
\hline Beta-lactams only & $5(7.46)$ \\
\hline Fluoroquinolones only & $3(4.48)$ \\
\hline Vancomycin ${ }^{\mathrm{e}}$ & $11(16.42)$ \\
\hline \multicolumn{2}{|l|}{ Oxygen support before sampling, n (\%) } \\
\hline High-flow nasal cannula only & $25(37.31)$ \\
\hline Mechanical ventilation & $41(61.19)$ \\
\hline Mechanical ventilation $\geq 2$ days, $n$ (\%) & $23(34.33)$ \\
\hline \multicolumn{2}{|l|}{ Severity variables at sampling } \\
\hline APACHE-II, mean \pm sd & $12.78 \pm 5.65$ \\
\hline PSI risk class IV-V, n (\%) & $41(61.19)$ \\
\hline
\end{tabular}




\begin{tabular}{|ll|}
\hline Variable & $\mathrm{N}=67$ \\
\hline CURB-65 risk score 3-5, $\mathrm{n}(\%)$ & $26(38.81)$ \\
\hline 7-category ordinal scale at sampling, $\mathrm{n}(\%)$ & $41(61.19)$ \\
\hline 5: Hospitalization, requiring HFNC and/or non-IMV & $25(37.31)$ \\
\hline 6: Hospitalization, requiring ECMO and/or IMV & $21(31.34)$ \\
\hline Decrease of 2 categories with 14 days & $5 \pm 4$ \\
\hline Days from illness onset to admission, median \pm IQR & $21 \pm 13$ \\
\hline Hours from admission to sampling ${ }^{f}$ median \pm IQR & \\
\hline ICU outcomes & $9 \pm 9.5$ \\
\hline ICU length of stay, days, median \pm IQR & $11(16.41)$ \\
\hline Death in ICU & $7(10.45)$ \\
\hline Day 14 mortality & \\
\hline Plasma Biomarker on admission, pg/ml, mean \pm sd & $5.64 \pm 12.6$ \\
\hline IL-4 & $336.30 \pm 725.60$ \\
\hline IL-6 & $61.50 \pm 144.24$ \\
\hline IL-8 & $169.01 \pm 498.12$ \\
\hline MIP-1beta & $1343.97 \pm 761.88$ \\
\hline VEGF-A & $142.34 \pm 156.14$ \\
\hline MMP-9 & \\
\hline a: Smokng & \\
\hline
\end{tabular}

a: Smoking status was grouped as nonsmokers, current smokers and former smokers (defined as the previous smokers who had quit at least 6 months before).

b: Two patients had positive urinary antigen for Streptococcus pneumoniae with negative detection in bronchoalveolar lavage fluid (BALF). Other microbiology results were totally obtained from the detection in BALF collected within 48 hours.

c: Atypical pathogens consisted of Legionella pneumophila and Mycoplasma pneumoniae.

d: Only 6 patients $(8.96 \%)$ had not received antibiotics before admitted to ICU. Among them, 2 patients received carbapenems and 4 patients received beta-lactams plus fluoroquinolones only.

e: Consisting of vancomycin, teicoplanin and linezolid.

$f:$ The order of performing bronchoscopy is based the time sequence of signing the bronchoscopy informed consent.

Abbreviation: IFD, invasive fungal disease; ARDS, acute respiratory distress syndrome; AKI, acute kidney injury; IQR, interquartile range; SD, standard deviation; ICU, intensive care unit. 


\section{Bacterial Detection Shaped The Lung Microbial Composition}

Plasma MIP-1 beta level (Bray-Curtis distance; $R^{2}=0.029 ; P=0.03$ ), procalcitonin level $\left(P C T R^{2}=0.029 ; P\right.$ $=0.026)$, complicated with $A K I\left(R^{2}=0.033 ; P=0.012\right)$, bacterial detection $\left(R^{2}=0.033 ; P=0.013\right)$ and the time from admission to sampling $\left(R^{2}=0.049 ; P=0.018\right.$ Fig. 2a-e) were significantly associated with the lung microbiota by univariate PERMANOVA. Age, baseline chronic respiratory diseases, antibiotic use, complicated with ARDS and septic shock (Fig. S1, Table S3) were not significantly associated with the lung microbiota.

We performed a multivariable analysis to assess the independent effect of individual factor. Smoking status $\left(R^{2}=0.036 ; P=0.185\right)$, time on ventilator $\left(R^{2}=0.04 ; P=0.097\right)$ before sampling and sputum production $\left(R^{2}=0.021 ; P=0.118 \mathrm{Fig} .2 f-h\right)$ whose $P$ value less than 0.2 were also included. Kappa test didn't show a severe multicollinearity problem among the eight factors ( $K=4.8$ Fig. $2 f)$. Bacterial detection showed the strongest independent relationship to the lung microbiota composition (multivariable PERMANOVA, $\left.R^{2}=0.033 ; p=0.018\right)$, followed by $A K I\left(R^{2}=0.032 ; p=0.011\right)$ and plasma MIP-1 beta level $\left(R^{2}=0.027 ; p=0.044\right)$.

\section{Bacteriology Results Influenced Lung Microbial Taxa}

Prevotellaceae family played the most important role in distinguishing bacterial detection positive from negative samples, followed by the pathogenic taxa, such as, families Moraxellaceae, Staphylococcaceae and Streptococcaceae (Fig. 3a). In the patients with positive bacteriology results, the mean relative abundance of families Prevotellaceae, Microbacteriaceae and Actinomycetaceae decreased while families Moraxellaceae, Staphylococcaceae and Streptococcaceae increased (Fig. 3a-b), which were in line with their high isolation rate of typical bacterial culture (Table 2). 16S rRNA gene sequencing was able to produce the same results as culture methods in $61 \%$ of patients (Table 2 ). However, there was no significant difference in the relative abundance of family Pseudomonadaceae between the two groups patients (Fig. 3a). The samples with bacteria detected had lower alpha diversity while the difference wasn't significant (Fig. 3d). 
Table 2

Comparison between bacterial detection and sequencing results

\begin{tabular}{|c|c|c|}
\hline $\begin{array}{l}M V \geq 2 \\
\text { days }^{a}\end{array}$ & Bacterial detection & $\begin{array}{l}\text { The most abundant ZOTU in } \\
\text { sequencing }\end{array}$ \\
\hline Yes & Acinetobacter baumannii & $\begin{array}{l}\text { ZOTU4_f_Streptococcaceae } \\
(38.43 \%)^{c}\end{array}$ \\
\hline Yes & Acinetobacter baumannii & ZOTU5_f_Moraxellaceae(87.82\%) \\
\hline Yes & $\begin{array}{l}\text { Acinetobacter baumannii Klebsiella } \\
\text { pneumoniae }\end{array}$ & ZOTU5_f_Moraxellaceae(37.71\%) \\
\hline Yes & Pseudomonas aeruginosa & $\begin{array}{l}\text { ZOTU1_f_Pseudomonadaceae } \\
(98.5 \%)\end{array}$ \\
\hline Yes & Pseudomonas aeruginosa & $\begin{array}{l}\text { ZOTU1_f_Pseudomonadaceae } \\
(83.84 \%)\end{array}$ \\
\hline Yes & Staphylococcus aureus & ZOTU18_f_Streptococcaceae (54.3\%) \\
\hline Yes & Staphylococcus aureus & $\begin{array}{l}\text { ZOTU10_f_Staphylococcaceae } \\
(29.84 \%)\end{array}$ \\
\hline No & Pseudomonas aeruginosa & $\begin{array}{l}\text { ZOTU18_f_Streptococcaceae } \\
(13.98 \%)\end{array}$ \\
\hline No & Pseudomonas aeruginosa & $\begin{array}{l}\text { ZOTU1_f_Pseudomonadaceae } \\
(69.33 \%)\end{array}$ \\
\hline No & Pseudomonas aeruginosa & $\begin{array}{l}\text { ZOTU1_f_Pseudomonadaceae } \\
(83.92 \%)\end{array}$ \\
\hline No & Pseudomonas aeruginosa & $\begin{array}{l}\text { ZOTU1_f_Pseudomonadaceae } \\
(36.14 \%)\end{array}$ \\
\hline No & Staphylococcus aureus & $\begin{array}{l}\text { ZOTU13_f_Corynebacteriaceae } \\
(15.91 \%)\end{array}$ \\
\hline No & Staphylococcus aureus & $\begin{array}{l}\text { ZOTU10_f_Staphylococcaceae } \\
(59.17 \%)\end{array}$ \\
\hline No & Streptococcus pneumoniae ${ }^{b}$ & ZOTU4_f_Streptococcaceae (98.02\%) \\
\hline
\end{tabular}

a: The patients had more than 2 days on ventilator were defined as "Yes" and less than 2 days or those never received mechanical ventilation were defined as "No".

b: Two patients had positive urinary antigen for Streptococcus pneumoniae with negative detection in bronchoalveolar lavage fluid (BALF).

c: The relative abundance of the most abundant ZOTU was shown.

The bold font represented that there were different results between bacterial detection and sequencing method.

Abbreviation: MV, mechanical ventilation. 


\begin{tabular}{|c|c|c|}
\hline $\begin{array}{l}M V \geq 2 \\
\text { days }^{a}\end{array}$ & Bacterial detection & $\begin{array}{l}\text { The most abundant ZOTU in } \\
\text { sequencing }\end{array}$ \\
\hline No & Streptococcus pneumoniae ${ }^{b}$ & $\begin{array}{l}\text { ZOTU1_f_-Pseudomonadaceae } \\
\text { (27.86\%) }\end{array}$ \\
\hline No & Acinetobacter baumannii & ZOTU5_f_Moraxellaceae(50.41\%) \\
\hline No & Klebsiella pneumoniae & ZOTU59_f_Bacteroidaceae (12.28\%) \\
\hline No & Escherichia coli & $\begin{array}{l}\text { ZOTU44_f_C Corynebacteriaceae } \\
(13.28 \%)\end{array}$ \\
\hline \multicolumn{3}{|c|}{$\begin{array}{l}\text { a: The patients had more than } 2 \text { days on ventilator were defined as "Yes" and less than } 2 \text { days or } \\
\text { those never received mechanical ventilation were defined as "No". }\end{array}$} \\
\hline \multicolumn{3}{|c|}{$\begin{array}{l}\text { b: Two patients had positive urinary antigen for Streptococcus pneumoniae with negative detection in } \\
\text { bronchoalveolar lavage fluid (BALF). }\end{array}$} \\
\hline \multicolumn{3}{|c|}{ c: The relative abundance of the most abundant ZOTU was shown. } \\
\hline \multicolumn{3}{|c|}{$\begin{array}{l}\text { The bold font represented that there were different results between bacterial detection and } \\
\text { sequencing method. }\end{array}$} \\
\hline
\end{tabular}

\section{Lung Microbiota Predicted Clinical Improvements}

The lung microbial community composition of the patients on admission wasn't associated with the clinical improvement (PERMANOVA; Bray-Curtis distance; $R^{2}=0.011 ; P=0.76$ ). Compared with the patients whose richness of baseline lung microbiota was higher than 200 , the multivariable-adjusted Hazard Ratio (HR) for the favorable prognosis in the lower Richness group patients was 0.17 and 0.16 , respectively ( $95 \% \mathrm{Cl}: 0.04-0.71, p=0.02 ; 95 \% \mathrm{Cl}: 0.03-0.88, p=0.04$ Fig. $4 a$, Table 3 ). The increased Shannon index predicted a faster decrease of 2 categories which was analyzed continuously (adjusted HR 1.54, 95\% Cl: $1.08-2.20, p=0.02$ ) or by organizing their value into ranges (adjusted HR $0.08,95 \% \mathrm{Cl}$ : $0.01-0.51, p=0.007$ Table 3, Fig. 4b, Table 3). Although the bacterial detection wasn't associated with clinical improvements, we found that every $1 \%$ increase in the relative abundance of families Prevotellaceae and Actinomycetaceae in the lung microbiota of the patients raised the probability for clinical improvements by $13 \%$ and $12 \%$ respectively (95\% Cl: $1.02-1.25, p=0.02 ; 95 \% \mathrm{Cl}: 1.03-1.21, p=$ 0.007 Table 3, Fig. 4c-d). However, families Moraxellaceae, Staphylococcaceae and Streptococcaceae were not associated with clinical improvements (Table 3, Fig. 4c-d). The proportions of patients with ARDS, septic shock, CURB-65 $\geq 3, P S I \geq I V$ were similar irrespective of the different level of alpha diversity or relative abundance of taxa (Table $\mathrm{S4}$ ). 
Table 3

Association of lung microbiota with clinical improvements in multivariable analysis.

\begin{tabular}{|c|c|c|c|}
\hline Variable & adjusted $\mathrm{HR}^{\mathrm{a}}$ & $95 \% \mathrm{Cl}$ & $P$ value \\
\hline \multicolumn{4}{|l|}{ Pathogen identified ${ }^{\mathrm{b}}$} \\
\hline Bacterial detection only & reference & & \\
\hline Viral-bacterial co-infection & 0.60 & $0.06-6.54$ & 0.67 \\
\hline Viral detection only & 1.82 & $0.48-6.92$ & 0.38 \\
\hline Others & 0.69 & $0.19-2.46$ & 0.57 \\
\hline Richness (continuous) & 1.01 & $1.00-1.02$ & 0.06 \\
\hline \multicolumn{4}{|l|}{ Richness (categorical) ${ }^{\mathrm{C}}$} \\
\hline$\geq 200$ & reference & & \\
\hline $199-150$ & 0.17 & $0.04-0.71$ & 0.02 \\
\hline$<150$ & 0.16 & $0.03-0.88$ & 0.04 \\
\hline Shannon (continuous) & 1.54 & $1.08-2.20$ & 0.02 \\
\hline \multicolumn{4}{|l|}{ Shannon (categorical) ${ }^{\mathrm{C}}$} \\
\hline$>4.5$ & reference & & \\
\hline $4.5-2$ & 0.38 & $0.11-1.28$ & 0.12 \\
\hline$<2$ & 0.08 & $0.01-0.51$ & 0.007 \\
\hline Prevotellaceae, $1 \%{ }^{\mathrm{d}}$ (continuous) & 1.13 & $1.02-1.25$ & 0.02 \\
\hline
\end{tabular}

a: Multiple cox regression analysis adjusted for sample season, plasma IL-8 level, CURB-65, APACHEII scores, presence of shock at sampling, oxygen index on admission, creatinine level and microbiology results.

b: Multiple cox regression analysis adjusted for sample season, plasma IL-8 level, CURB-65, APACHEII scores, presence of shock at sampling, oxygen index on admission, creatinine level.

c: We turned the continuous variables into categorical variables by organizing their value into ranges. Firstly, according to the value of the variable, 67 samples were put in order. Secondly, we divided them into 10 groups evenly. Finally, we combined the neighboring groups that had the similar probability for the event happened.

d: $1 \%$ referred to that the probability for clinical improvements of every $1 \%$ increase in the relative abundance of taxa.

Abbreviation: $\mathrm{HR}$, hazard ratios; $\mathrm{Cl}$, confidence interval. 


\begin{tabular}{|c|c|c|c|}
\hline Variable & adjusted $\mathrm{HR}^{\mathrm{a}}$ & $95 \% \mathrm{Cl}$ & $P$ value \\
\hline$>2.4$ & \multicolumn{3}{|l|}{ reference } \\
\hline $2.4-0.3$ & 0.37 & $0.10-1.38$ & 0.14 \\
\hline$<0.3$ & 0.08 & $0.01-0.39$ & 0.002 \\
\hline Actinomycetaceae, $1 \%$ (continuous) & 1.12 & $1.03-1.21$ & 0.007 \\
\hline \multicolumn{4}{|l|}{ Actinomycetaceae, \% (categorical) ${ }^{\mathrm{C}}$} \\
\hline$>0.5$ & \multicolumn{3}{|l|}{ reference } \\
\hline $0.5-0.005$ & 0.37 & $0.11-1.21$ & 0.10 \\
\hline$<0.005$ & 0.06 & $0.01-0.33$ & 0.001 \\
\hline Moraxellaceae, $1 \%$ (continuous) & 1.04 & $0.98-1.09$ & 0.17 \\
\hline Staphylococcaceae, $1 \%$ (continuous) & 0.95 & $0.89-1.02$ & 0.19 \\
\hline Streptococcaceae,1\% (continuous) & 1.01 & $0.98-1.04$ & 0.62 \\
\hline Pseudomonadaceae, $1 \%$ (continuous) & 0.99 & $0.97-1.00$ & 0.09 \\
\hline Enterobacteriaceae, $1 \%$ (continuous) & 0.95 & $0.82-1.10$ & 0.49 \\
\hline \multicolumn{4}{|c|}{$\begin{array}{l}\text { a: Multiple cox regression analysis adjusted for sample season, plasma IL-8 level, CURB- } 65 \text {, APACHEII } \\
\text { scores, presence of shock at sampling, oxygen index on admission, creatinine level and microbiology } \\
\text { results. }\end{array}$} \\
\hline \multicolumn{4}{|c|}{$\begin{array}{l}\text { b: Multiple cox regression analysis adjusted for sample season, plasma IL-8 level, CURB-65, APACHEII } \\
\text { scores, presence of shock at sampling, oxygen index on admission, creatinine level. }\end{array}$} \\
\hline \multicolumn{4}{|c|}{$\begin{array}{l}\text { c: We turned the continuous variables into categorical variables by organizing their value into ranges. } \\
\text { irstly, according to the value of the variable, } 67 \text { samples were put in order. Secondly, we divided them } \\
\text { into } 10 \text { groups evenly. Finally, we combined the neighboring groups that had the similar probability for } \\
\text { the event happened. }\end{array}$} \\
\hline \multicolumn{4}{|c|}{$\begin{array}{l}\text { d: } 1 \% \text { referred to that the probability for clinical improvements of every } 1 \% \text { increase in the relative } \\
\text { abundance of taxa. }\end{array}$} \\
\hline Abbreviation: $\mathrm{HR}$, hazard ratios; $\mathrm{Cl}$, con & & & \\
\hline
\end{tabular}

\section{Discussion}

The core findings of our study were that bacterial detection had the strongest independent relationship with the lung microbiota composition on patients' admission, followed by AKI and plasma MIP-1beta level. The increased alpha diversity and the enrichment of families Prevotellaceae and Actinomycetaceae in the lung microbiota were associated with clinical improvements. 
In this study, the positive bacterial detection on admission was found to be the most important factor related to patients' lung microbiota. The bacteria that could be identified by culture-based methods might have a higher load in the lower respiratory tract[22]. Therefore, 16S rRNA gene sequencing revealed that the lung microbiota dominated by the corresponding taxa in the patients with positive bacteriology results (such as Acinetobacter baumannii and Staphylococcus aureus). Although the results of 16S rRNA and culture-based methods were consistent in terms of dominant bacteria, the results related to Pseudomonas aeruginosa differed. The relative abundance of family Pseudomonadaceae wasn't significantly different between the bacteriology-positive and bacteriology-negative groups despite the high isolation rate (33.33\%) of Pseudomonas aeruginosa. P. aeruginosa is one of the main nosocomial pathogens in ICU. The risk factors for $P$. aeruginosa acquisition consisted of antibiotics pressure, mechanical ventilation use, length of hospitalization, cross-transmission among patients and medical staffs and environmental factors, such as contamination of water tap[23-25]. All of our patients had received antimicrobial treatment and more than half were exposed to mechanical ventilation before bronchoscopy. They might have similar opportunity to obtain $P$. aeruginosa colonization under the ICU status. Although 16S rRNA gene sequencing had a low accuracy for pathogen identification, it could capture all the bacterial information, including fastidious bacteria in the respiratory tract, which might explain the different results between culture-dependent and culture-independent analysis in our study[26, 27].

After adjusted for the microbiology results and severity of illness, multivariate analysis showed that clinical improvements were associated with increased relative abundance of family Prevotellaceae and Actinomycetaceae. It was found that relative abundance of Prevotella was inversely associated with airway inflammation in cystic fibrosis patients[28]. An increase in the abundance of Prevotella in nose/throat microbiota was related to lower susceptibility to influenza A infection[29]. Another set of researches showed that the enrichment of lung microbiota with Prevotella enhanced the level of BALF inflammatory cytokines, and was associated with the development of asthma in children and ARDS in severe patients[7, 30, 31]. The conclusions appeared to be inconsistent. However, the reduction in abundance of Prevotella was observed at the initial stage of chronic pulmonary inflammation in mice[32]. The causal relationship between Prevotella and the diseases couldn't be clearly inferred from these association studies[33]. Besides, families Prevotellaceae and Actinomycetaceae usually resided in healthy the respiratory tract or oral cavity[30, 34, 35]. Earlier study reported that the commensal bacteria (commensal Prevotella spp and Actinomyces spp) could induce weak activation of human dendritic cells compared with pathogenic species (Haemophillus spp. and Moraxella spp)[36]. While co-culture experiments observed that Prevotella spp. were able to inhibit Haemophillus influenzaeinduced IL-12p70 in dendritic cells[36]. Conclusively, we thought that Prevotellaceae and Actinomycetaceae served as commensal colonization and might promote the recovery of the lung from infection in our study.

The antibiotic use was also proved to decrease the airway microbial diversity in COPD patients[37]. However, the type of antibiotic use before sampling was not associated with the lung microbiota in our study, consistent with the result of a study in intubation patients[9]. As all of our patients had received antibiotics before bronchoscopy, we couldn't exclude the possibility of an association between the 
antibiotics and the lung microbiota. Besides, the lung microbiota of our patients wasn't clustered by the presence of ARDS or septic shock at sampling. Unlike the report in two previous published studies, the relative abundance of family Enterobacteriaceae had no association with the severity of pneumonia or clinical outcomes[7, 8]. In fact, those studies primarily focused on the patients with ARDS caused by severe trauma, cardiac arrest or cerebral vascular events. In Robert P. Dickson et al.'s study, the pneumonia or sepsis patients merely accounted for $13 \%$ (12/91) while in Ariane R. Panzer's study, the ARDS in all patients was caused by trauma[7, 8]. Thus, the conclusions of the studies might not be applied to the SCAP patients.

Our study has several limitations. Firstly, the lung microbiota analysis was only performed on ICU admission. The variation of the lung microbiota during the patients' hospitalization and the association between that with the clinical improvements could not be analyzed. Secondly, the sample size of the patients was small. Finally, we couldn't explain that the relative abundance of Families Prevotellaceae and Actinomycetaceae or the alpha diversity was not associated with the severity of pneumonia. It seemed that high diversity and the presence of commensal colonization in the lung only suggested the ability to recover from infection. Further studies are needed to explore whether the lung microbiota play a role in the pathogenesis of pneumonia.

\section{Conclusions}

Conclusively, we found that the bacterial detection and patients' intrinsic factors were associated with the lung microbiota. The lung microbiota on admission was predictive of clinical improvements.

\section{Abbreviations}

SCAP, severe community-acquired pneumonia; ICU, intensive care unit;

BALF, bronchoalveolar lavage fluid; PERMANOVA, Permutational multivariate analysis of variance; AKI, acute kidney injury; ARDS, acute respiratory distress syndrome; HR, hazard ratios; $\mathrm{Cl}$, confidence interval.

\section{Declarations}

\section{Ethics approval and consent to participate}

Our study was conducted in China-Japan Friendship Hospital, Beijing, China and approved by the ChinaJapan Friendship Hospital Ethics Committee (Approval No. 2018-20-K15). It was conducted in accordance with the amended Declaration of Helsinki. All subjects provided written consents.

\section{Consent for publication}

Not applicable. 
The sequence data have been deposited in the NCBI Sequence Read Archive under accession PRJNA616057. (https://www.ncbi.nlm.nih.gov/bioproject/PRJNA616057). Feature, taxonomy, metadata tables and a reproducible workflow of the analysis are available for download at https://github.com/Zoey-Du/SCAP_microbiota.

\section{Competing interests}

The authors declare that they have no competing interests.

\section{Funding}

This study was funded by National Key Research and Development Program of China (2017YFC1309301), the CAMS Innovation Fund for Medical Sciences (CIFMS 2018-I2M-1-003), the National Science Grant for Distinguished Young Scholars (81425001/H0104), the Ministry of Science and Technology of China (2017ZX10103004).

\section{Authors' contributions}

B.C., Y.L. and B.L. conceived the idea. S.D., B.L., X.Z. and B.C. finished the protocol. S.D., The samples were collected by B.L., Z.X., D.Y., Z.L., and Y.D. S.D., L.S., C.W., X.L. and X.Z. performed the laboratory testing and data analysis. S.D., L.S. and B.C. contributed to the interpretation of the data. S.D., B.L., L.S. and B.C. drafted the manuscript. Y.L., B.L. and B.C. revised the manuscripts. All authors approved the manuscript.

\section{Acknowledgements}

Not applicable.

\section{References}

1. Espinoza R, Silva J, Bergmann A, de Oliveira MU, Calil FE, Santos RC, Salluh J. Factors associated with mortality in severe community-acquired pneumonia: A multicenter cohort study. J CRIT CARE. 2019;50:82-6.

2. Phua J, Dean NC, Guo Q, Kuan WS, Lim HF, Lim TK. Severe community-acquired pneumonia: timely management measures in the first 24 hours. CRIT CARE 2016, 20(1).

3. Li HY, Guo Q, Song WD, Zhou YP, Li M, Chen XK, Liu H, Peng HL, Yu HQ, Chen X, et al. Mortality among severe community-acquired pneumonia patients depends on combinations of 2007 IDSA/ATS minor criteria. INT J INFECT DIS. 2015;38:141-5.

4. Charlson ES, Bittinger K, Haas AR, Fitzgerald AS, Frank I, Yadav A, Bushman FD, Collman RG. Topographical Continuity of Bacterial Populations in the Healthy Human Respiratory Tract. AM J RESP CRIT CARE. 2011;184(8):957-63. 
5. Brown RL, Sequeira RP, Clarke TB. The microbiota protects against respiratory infection via GM-CSF signaling. NAT COMMUN. 2017;8(1):1512.

6. Pettigrew MM, Gent JF, Kong Y, Wade M, Gansebom S, Bramley AM, Jain S, Arnold SLR, McCullers JA. Association of sputum microbiota profiles with severity of community-acquired pneumonia in children. BMC INFECT DIS 2016, 16(1).

7. Panzer AR, Lynch SV, Langelier C, Christie JD, McCauley K, Nelson M, Cheung CK, Benowitz NL, Cohen MJ, Calfee CS. Lung Microbiota Is Related to Smoking Status and to Development of Acute Respiratory Distress Syndrome in Critically III Trauma Patients. AM J RESP CRIT CARE. 2018;197(5):621-31.

8. Dickson RP, Schultz MJ, van der Poll T, Schouten LR, Falkowski NR, Luth JE, Sjoding MW, Brown CA, Chanderraj R, Huffnagle GB, et al: Lung Microbiota Predict Clinical Outcomes in Critically III Patients. AM J RESP CRIT CARE 2020.

9. Zakharkina T, Martin-Loeches I, Matamoros S, Povoa P, Torres A, Kastelijn JB, Hofstra JJ, de Wever B, de Jong $\mathrm{M}$, Schultz $\mathrm{MJ}$, et al. The dynamics of the pulmonary microbiome during mechanical ventilation in the intensive care unit and the association with occurrence of pneumonia. THORAX. 2017;72(9):803-10.

10. Dickson RP, Erb-Downward JR, Huffnagle GB. Towards an ecology of the lung: new conceptual models of pulmonary microbiology and pneumonia pathogenesis. The Lancet Respiratory Medicine. 2014;2(3):238-46.

11. Dickson RP, Erb-Downward JR, Martinez FJ, Huffnagle GB. The Microbiome and the Respiratory Tract. ANNU REV PHYSIOL. 2016;78(1):481-504.

12. Dickson RP. The microbiome and critical illness. The Lancet Respiratory Medicine. 2016;4(1):59-72.

13. Mandell LA, Wunderink RG, Anzueto A, Bartlett JG, Campbell GD, Dean NC, Dowell SF, File TM, Musher DM, Niederman MS, et al. Infectious Diseases Society of America/American Thoracic Society Consensus Guidelines on the Management of Community-Acquired Pneumonia in Adults. CLIN INFECT DIS. 2007;44(Supplement_2):27-72.

14. Cao B, Wang K, Wang J, Wang J, Wang Y, Wang S, Wang C, Wen D, Liu X, Liu W, et al. A Trial of Lopinavir-Ritonavir in Adults Hospitalized with Severe Covid-19. The New England Journal of Medicine. 2020;382(19):1787-99.

15. Wang Y, Fan G, Horby P, Hayden F, Li Q, Wu Q, Zou X, Li H, Zhan Q, Wang C, et al: Comparative Outcomes of Adults Hospitalized With Seasonal Influenza A or B Virus Infection: Application of the 7Category Ordinal Scale. OPEN FORUM INFECT DI 2019, 6(3).

16. Wang Y, Zhang D, Du G, Du R, Zhao J, Jin Y, Fu S, Gao L, Cheng Z, Lu Q, et al. Remdesivir in adults with severe COVID-19: a randomised, double-blind, placebo-controlled, multicentre trial. The Lancet. 2020;395(10236):1569-78.

17. Zhou F, Wang Y, Liu Y, Liu X, Gu L, Zhang X, Pu Z, Yang G, Liu B, Nie Q, et al: Disease severity and clinical outcomes of community-acquired pneumonia caused by non-influenza respiratory viruses in 
adults: a multicentre prospective registry study from the CAP-China Network. EUR RESPIR J 2019, $54(2)$.

18. Ferguson ND, Fan E, Camporota L, Antonelli M, Anzueto A, Beale R, Brochard L, Brower R, Esteban A, Gattinoni $L$, et al. The Berlin definition of ARDS: an expanded rationale, justification, and supplementary material. Intensive Care Med. 2012;38(10):1573-82.

19. Singer M, Deutschman CS, Seymour CW, Shankar-Hari M, Annane D, Bauer M, Bellomo R, Bernard GR, Chiche JD, Coopersmith CM, et al. The Third International Consensus Definitions for Sepsis and Septic Shock (Sepsis-3). JAMA. 2016;315(8):801-10.

20. Khwaja A. KDIGO clinical practice guidelines for acute kidney injury. Nephron Clin Pract. 2012;120(4):c179-84.

21. Donnelly JP, Chen SC, Kauffman CA, Steinbach WJ, Baddley JW, Verweij PE, Clancy CJ, Wingard JR, Lockhart SR, Groll AH, et al: Revision and Update of the Consensus Definitions of Invasive Fungal Disease From the European Organization for Research and Treatment of Cancer and the Mycoses Study Group Education and Research Consortium. CLIN INFECT DIS 2019.

22. Kelly BJ, Imai I, Bittinger K, Laughlin A, Fuchs BD, Bushman FD, Collman RG. Composition and dynamics of the respiratory tract microbiome in intubated patients. MICROBIOME 2016, 4(1).

23. Venier AG, Leroyer C, Slekovec C, Talon D, Bertrand X, Parer S, Alfandari S, Guerin JM, Megarbane B, Lawrence $\mathrm{C}$, et al. Risk factors for Pseudomonas aeruginosa acquisition in intensive care units: a prospective multicentre study. J HOSP INFECT. 2014;88(2):103-8.

24. Hoang S, Georget A, Asselineau J, Venier A, Leroyer C, Rogues AM, Thiébaut R. Risk factors for colonization and infection by Pseudomonas aeruginosa in patients hospitalized in intensive care units in France. PLOS ONE. 2018;13(3):e193300.

25. Bachta KER, Allen JP, Cheung BH, Chiu C, Hauser AR. Systemic infection facilitates transmission of Pseudomonas aeruginosa in mice. NAT COMMUN2020, 11(1).

26. Hilton SK, Castro-Nallar E, Pérez-Losada M, Toma I, McCaffrey TA, Hoffman EP, Siegel MO, Simon GL, Johnson WE, Crandall KA. Metataxonomic and Metagenomic Approaches vs. Culture-Based Techniques for Clinical Pathology. FRONT MICROBIOL 2016, 7.

27. Emonet S, Lazarevic V, Leemann Refondini C, Gaïa N, Leo S, Girard M, Nocquet Boyer V, Wozniak H, Després $L$, Renzi $G$, et al. Identification of respiratory microbiota markers in ventilator-associated pneumonia. INTENS CARE MED. 2019;45(8):1082-92.

28. Zemanick ET, Wagner BD, Robertson CE, Ahrens RC, Chmiel JF, Clancy JP, Gibson RL, Harris WT, Kurland G, Laguna TA, et al. Airway microbiota across age and disease spectrum in cystic fibrosis. EUR RESPIR J. 2017;50(5):1700832.

29. Tsang TK, Lee KH, Foxman B, Balmaseda A, Gresh L, Sanchez N, Ojeda S, Lopez R, Yang Y, Kuan G, et al: Association Between the Respiratory Microbiome and Susceptibility to Influenza Virus Infection. CLIN INFECT DIS 2019.

30. Segal LN, Clemente JC, Tsay JJ, Koralov SB, Keller BC, Wu BG, Li Y, Shen N, Ghedin E, Morris A, et al: Enrichment of the lung microbiome with oral taxa is associated with lung inflammation of a Th17 
phenotype. NAT MICROBIOL 2016, 1(5).

31. Thorsen J, Rasmussen MA, Waage J, Mortensen M, Brejnrod A, Bønnelykke K, Chawes BL, Brix S, Sørensen SJ, Stokholm J, et al: Infant airway microbiota and topical immune perturbations in the origins of childhood asthma. NAT COMMUN2019, 10(1).

32. Yadava K, Pattaroni C, Sichelstiel AK, Trompette A, Gollwitzer ES, Salami O, von Garnier C, Nicod LP, Marsland BJ. Microbiota Promotes Chronic Pulmonary Inflammation by Enhancing IL-17A and Autoantibodies. AM J RESP CRIT CARE. 2016;193(9):975-87.

33. Huffnagle GB, Dickson RP, Lukacs NW. The respiratory tract microbiome and lung inflammation: a two-way street. MUCOSAL IMMUNOL. 2017;10(2):299-306.

34. Morris A, Beck JM, Schloss PD, Campbell TB, Crothers K, Curtis JL, Flores SC, Fontenot AP, Ghedin E, Huang $L$, et al. Comparison of the Respiratory Microbiome in Healthy Nonsmokers and Smokers. AM J RESP CRIT CARE. 2013;187(10):1067-75.

35. Li X, Sun Y, An Y, Wang R, Lin H, Liu M, Li S, Ma M, Xiao C. Air pollution during the winter period and respiratory tract microbial imbalance in a healthy young population in Northeastern China. ENVIRON POLLUT. 2019;246:972-9.

36. Larsen JM, Steen-Jensen DB, Laursen JM, Sondergaard JN, Musavian HS, Butt TM, Brix S. Divergent pro-inflammatory profile of human dendritic cells in response to commensal and pathogenic bacteria associated with the airway microbiota. PLOS ONE. 2012;7(2):e31976.

37. Wang Z, Bafadhel M, Haldar K, Spivak A, Mayhew D, Miller BE, Tal-Singer R, Johnston SL, Ramsheh MY, Barer MR, et al. Lung microbiome dynamics in COPD exacerbations. EUR RESPIR J. 2016;47(4):1082-92.

\section{Figures}




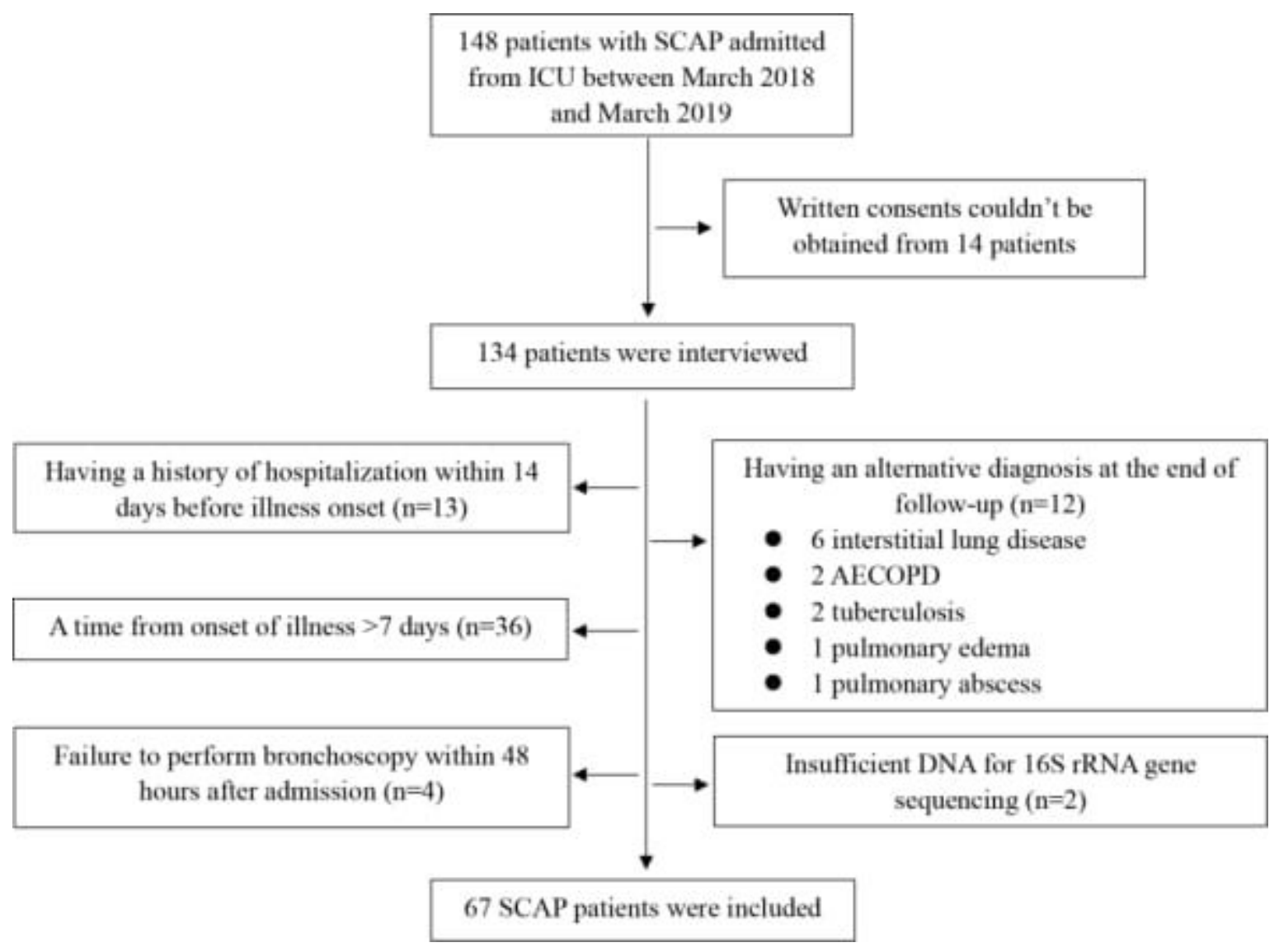

\section{Figure 1}

Study flow chart *The patients whose time of disease less than 14 days while condition exacerbation within 7 days before admission to ICU were also included. Abbreviation: SCAP, severe community acquired pneumonia; ICU, intensive care unit; AECOPD, acute exacerbation of chronic obstructive pulmonary disease. 

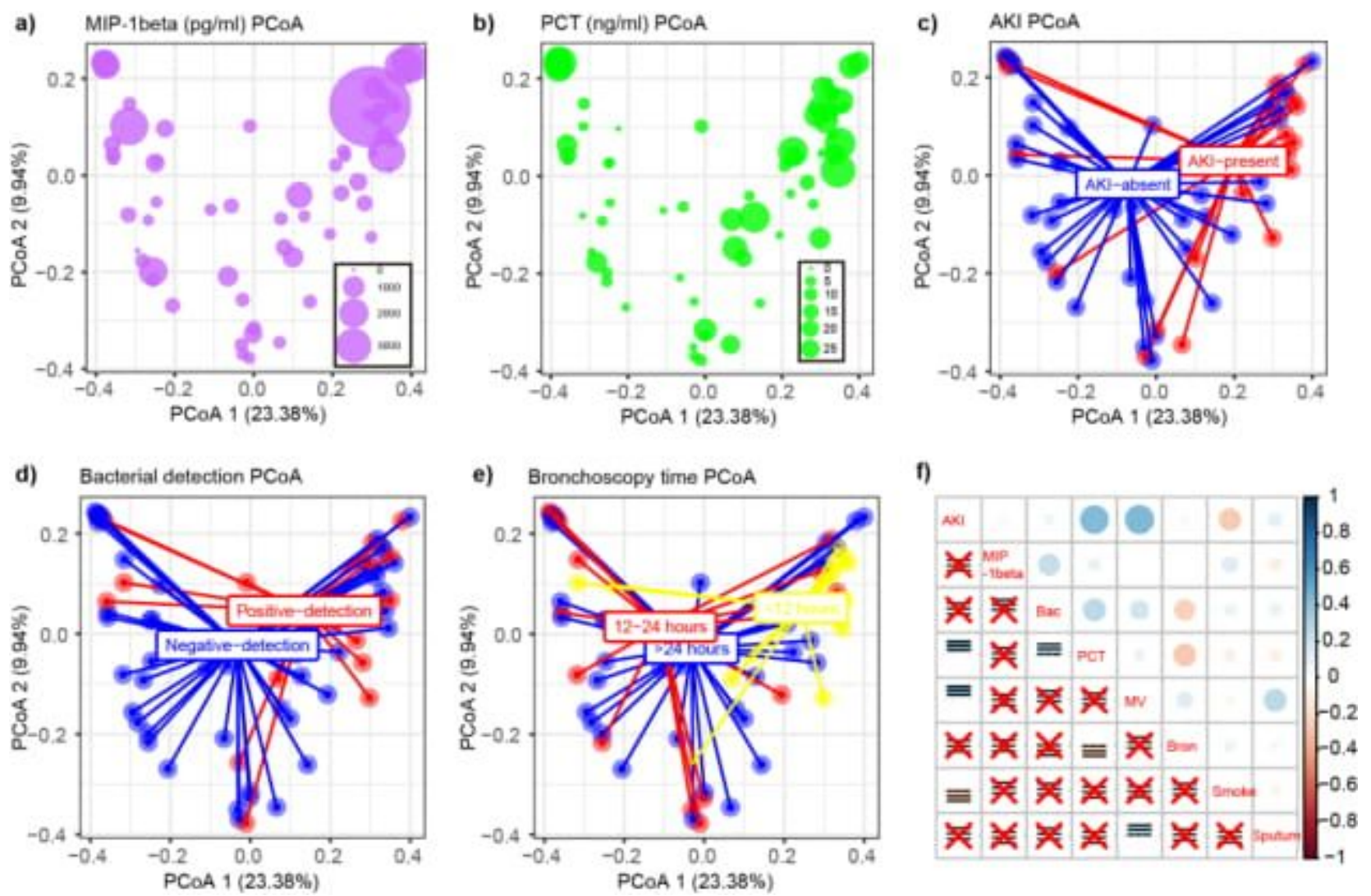

Figure 2

Factors associated with the lung microbiota Principal Coordinate Analysis (PCoA) plot based on BrayCurtis distance revealed that the lung microbial community composition was significantly associated with the concentration of MIP-1 beta (a) and procalcitonin (PCT b). The lung microbiota was associated with the presence of acute kidney injury (AKI) on admission (c), bacterial detection (Bac d) and the time from admission to sampling (Bron e). The co-association between the 8 clinical factors was calculated by Pearson correlation analysis (f). The circles were proportional to the absolute value of the correlation coefficients. The squares represented the $P$ values. " $X$ " represented the $P$ values were larger than 0.05 . 
a)

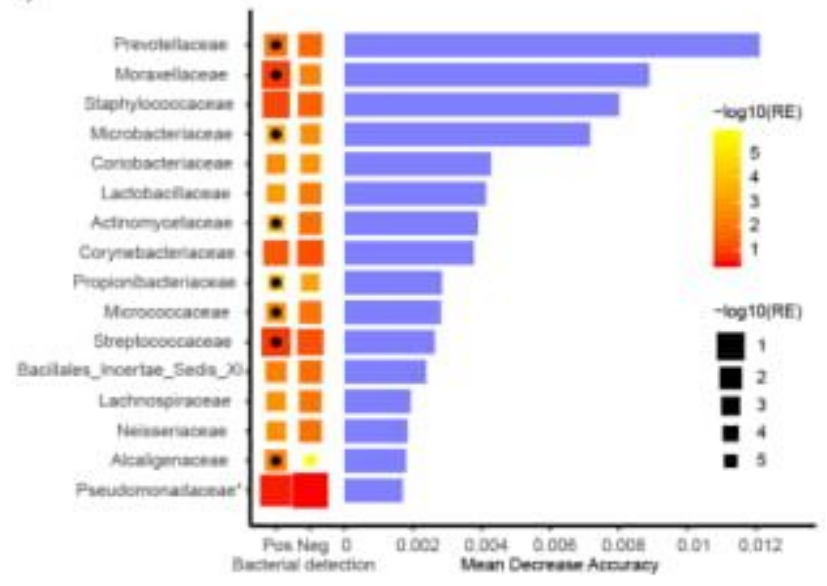

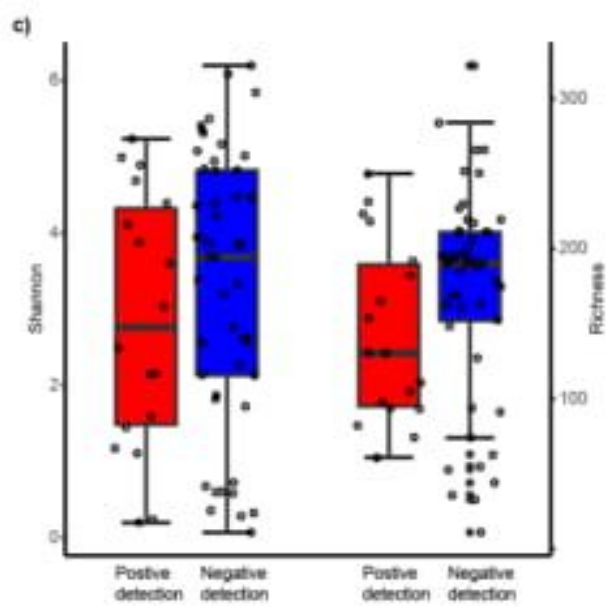

b)

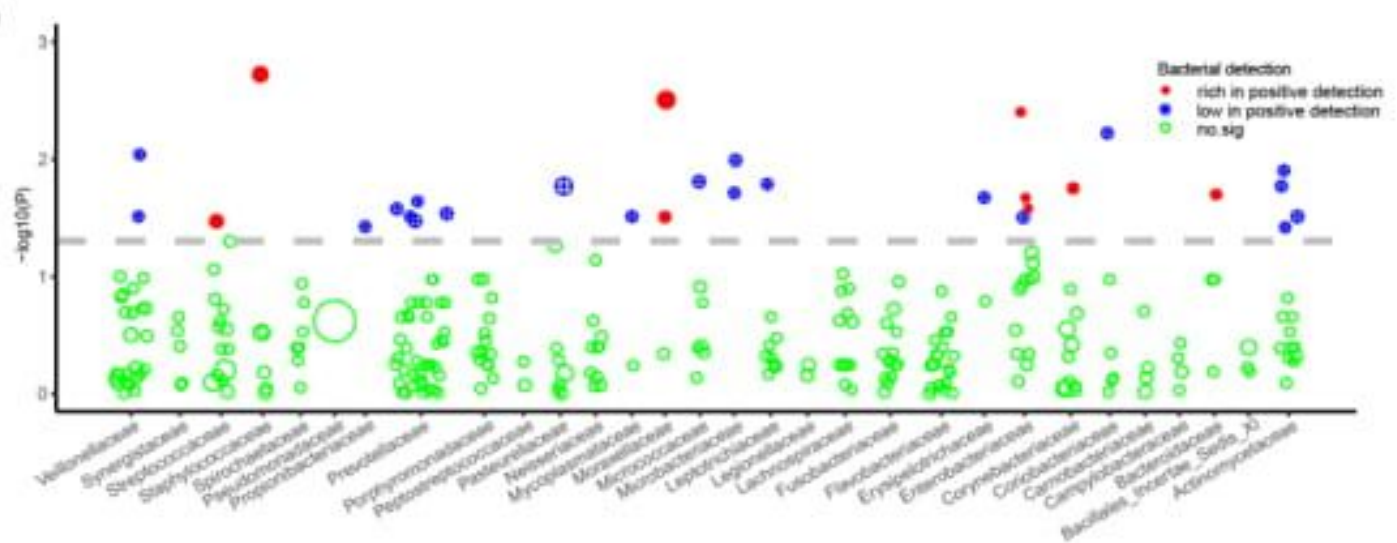

Figure 3

The influence of bacteriology results on lung microbiota The top 15 biomarker taxa associated with bacterial detection (a). The black points represented there were significantly different in the relative abundance of the species between the two groups. The color and size of the squares were proportional to the value of $\log 10$ (mean relative abundance). *: The variation of the relative abundance in family Pseudomonadaceae that was not included in the top 15 was shown. (b) The positions of the ZOTUs were determined by the value of -log10(P value from the comparison of the mean relative abundance in ZOTUs between the groups). The size of circles was proportional to the relative abundance of the ZOTUs in microbiota. (c) The boxplots represent the diversity measures (Shannon index and Richness) for the patients (center line, median; box limits, \pm interquartile range; whisker limits, maximum/minimum). 
a)

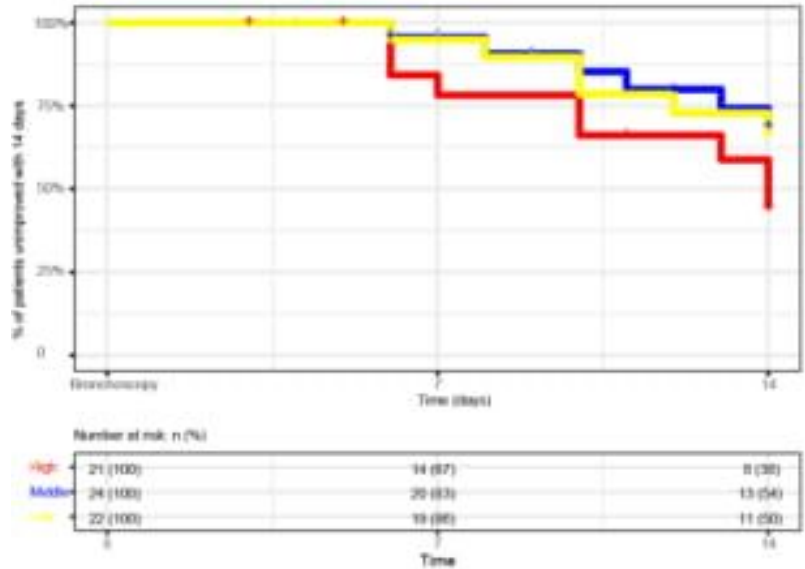

c)

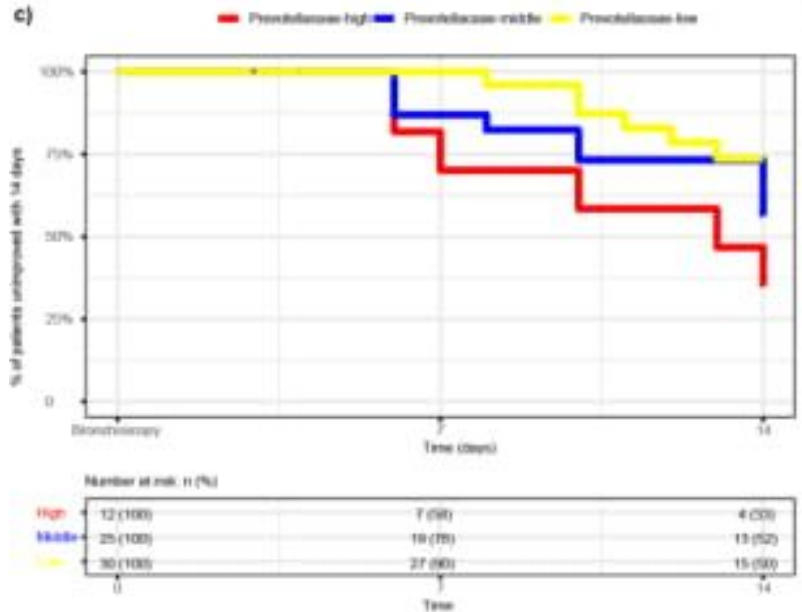

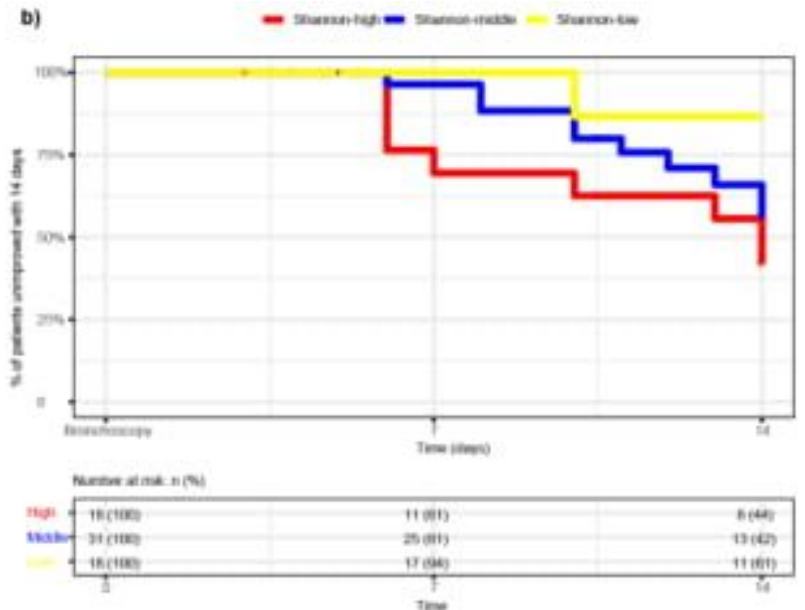

d)

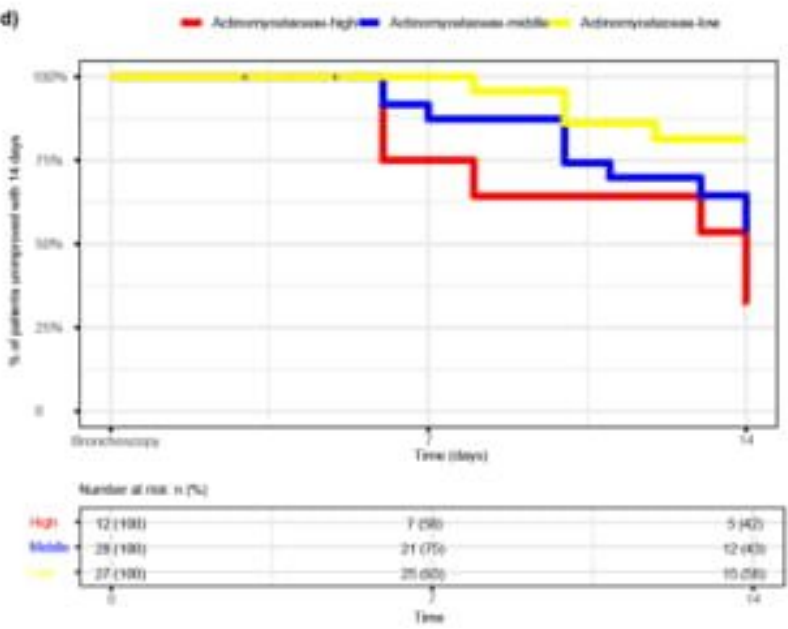

Figure 4

Lung microbiota predicting clinical improvements Survival curves were plotted using the Kaplan-Meier method. The primary endpoint was the rate of clinical improvements (defined as a decrease of 2 categories and above on a 7-category ordinal scale) within 14 days following bronchoscopy. Richness (a), Shannon index (b), the relative abundance of families Prevotellaceae (c) and Flavobacteriaceae (d) were protective of the clinical improvements after adjusted for sample season, plasma IL-8 level, CURB65 , APACHEll scores, presence of shock at sampling, oxygen index on admission, creatinine level and microbiology results. The categorical cut-offs were consistent with those in Table 3

\section{Supplementary Files}

This is a list of supplementary files associated with this preprint. Click to download.

- supplement.docx 